\title{
Thermal boundary conditions around thin Pt wires under the current supply and its application for Joule heat welding of wires having various diameters
}

\author{
Hironori TOHMYOH* and Kanta YAMAGUCHI* \\ *Department of Finemechanics, Tohoku University \\ Aoba 6-6-01, Aramaki, Aoba-ku, Sendai 980-8579, Japan \\ E-mail: tohmyoh@ism.mech.tohoku.ac.jp
}

Received: 19 November 2018; Revised: 31 January 2019; Accepted: 4 February 2019

\begin{abstract}
This paper treats the Joule heat welding of thin Pt wires having various diameters. Joining of fine-scale materials, such as micro- or nanowires, has been essential to create advanced materials systems. Joule heat welding is one of the suitable way for this purpose. It has been reported that thin wires were welded together under the supply of constant direct current, and the welding condition was sensitive to the thermal boundary conditions around the wires under the current supply, which was affected by the heat transfer from the wire surface to the ambient and the heat conduction at the ends for the current supply. The diameters of thin Pt wires were 0.8-20 $\mu \mathrm{m}$, and the current required to cut the wire by Joule heating was investigated. The temperature of the wire having the smaller diameter was easily increased by Joule heating, and this became remarkable with increasing the slenderness ratio, i.e., the ratio of the diameter to the length for current supply. An index related to the temperature of the wire under the current supply was introduced to describe the thermal boundary conditions around the wire. The behavior of the index against the diameter was approximated by the exponential functions, and the thermal boundary conditions around the wires having various diameters became predictable. Finally, the validity of the thermal boundary conditions determined and predicted in this study was verified by conducting the experiments to cut and weld the wires by Joule heating.
\end{abstract}

Keywords : Joule heating, Welding, Thin wire, Diameter, Thermal boundary conditions

\section{Introduction}

Micro- and nanomaterials have excellent physical properties and unique geometrical features, and therefore, such fine-scale materials are expected to be used as essential elements in advanced materials systems. For this purpose, expanding the techniques for freely using fine-scale materials have been essential. Among such techniques, joining of fine-scale materials is vital. Various techniques to join fine-scale materials, such as metallic or semiconducting nanomaterials (Kim, et al., 2005; Xu, et al., 2005; Tohmyoh, et al., 2007; Lu, et al., 2010; Tan and Wang, 2013; Peng, et al., 2013; Vafaei, et al., 2014; Ma, et al., 2016) and carbon nanotube (Hirayama, et al., 2001; Dong, et al., 2007; Jin, et al., 2007) have been reported. Electron beam (Xu, et al., 2005), laser beam (Kim, et al., 2005; Hu, et al., 2012; Dai, et al., 2016), or light (Garnett, et al., 2012) is used to melt a fine-scale materials for joining. Also, nano-volume solder is used to realize the soldering of nanowires (Peng, et al., 2009).

Joule heating is effective to increase the temperature of fine-scale materials having smaller heat capacity (Hummelgård, et al., 2010), and is used to join such materials (Hirayama, et al., 2001; Tohmyoh, et al., 2007; Song, et al., 2014). It has been reported that thin Pt wires are welded together in self-completed matter under the supply of constant direct current (Tohmyoh and Fukui, 2009). Tip of a thin wire was welded to the side of another thin wire (Fukui and Tohmyoh, 2011) or the surface of the substrate (Tohmyoh and Fujimori, 2014). Thin wires having dissimilar diameter (Fujimori and Tohmyoh, 2013) and dissimilar metal wires were also welded together by Joule heating (Tohmyoh and Sunagawa, 2015). In case of joining two thin wires together, temperature of the wire under the current supply is important. 
However, knowing the temperature is not so easy task because the thermal boundary conditions around the wire is usually unknown. In the previous studies, the thermal boundary conditions around the wire under the current supply was described by an index (Tohmyoh, 2009), and the index was determined experimentally by cutting the wire with Joule heating.

In this paper, we investigated the effect of the diameters of thin wires on the thermal boundary conditions around the wires under the current supply. Thin Pt wires having the diameters of $0.8,5$, and $20 \mu \mathrm{m}$ were treated in this study, and these were cut at various lengths for current supply. At the same slenderness ratio, which is the ratio of the diameter to the length for current supply, the temperature of thinner wire was easily raised by Joule heating compared with that of thicker one. Thermal boundary conditions around the wire was described by an index determined from the current required to cut the wire, and the behavior of the index against the diameter was approximated by an exponential function. Thermal boundary conditions around the wire having various diameters were predicted by interpolation and extrapolation, and the validity of the prediction was verified by conducting the experiments in different diameters. The wires having various diameters were welded, and the welding condition were summarized by considering the thermal boundary conditions around the wires.

\section{An index to describe the thermal boundary conditions around the wire}

Let us consider the electro-thermal problem, where the constant direct current, $I$, is supplied to a thin wire with a pair of the current terminals, see Fig. 1(a). In this case, the temperature of the wire takes its maximum value at the middle of two current terminals, and the wire is cut at the middle provided that the maximum temperature reaches the melting point of the wire. The current required to cut the wire, $\left(I_{\mathrm{C}}\right)_{\mathrm{EXP}}$, is related to the heat transfer from the wire surface to the ambient and the heat conduction at the ends for the current supply, and the temperature in the wire is affected by such thermal boundary conditions around the wire. The thermal boundary conditions around the wire under the current supply can be described as (Tohmyoh, 2009)

$$
f=\frac{\left(I_{\mathrm{C}}\right)_{0}}{\left(I_{\mathrm{C}}\right)_{\mathrm{EXP}}}
$$

where $\left(I_{\mathrm{C}}\right)_{0}$ is the current required to cut the wire under the thermal boundary conditions, which are described as follows: There is no heat transfer from the wire surface, and the temperature at the ends for current supply is constant at the ambient temperature, $T_{0}$, see Fig. $1(\mathrm{~b})$. The value $\left(I_{\mathrm{C}}\right)_{0}$ can be determined analytically by solving the heat conduction equation under the thermal boundary conditions, see Appendix, and it can be expressed as:

$$
\left(I_{\mathrm{C}}\right)_{0}=\frac{A}{L} \sqrt{8 K \sigma\left(T_{\mathrm{M}}-T_{0}\right)}
$$

where $A\left(=\pi d^{2} / 4\right)$ is the cross-sectional area of the wire, $d$ the diameter of the wire, $L$ is the length of the segment for the current supply, $K$ is the thermal conductivity, $\sigma$ the electrical conductivity, and $T_{\mathrm{M}}$ melting point of the wire. The
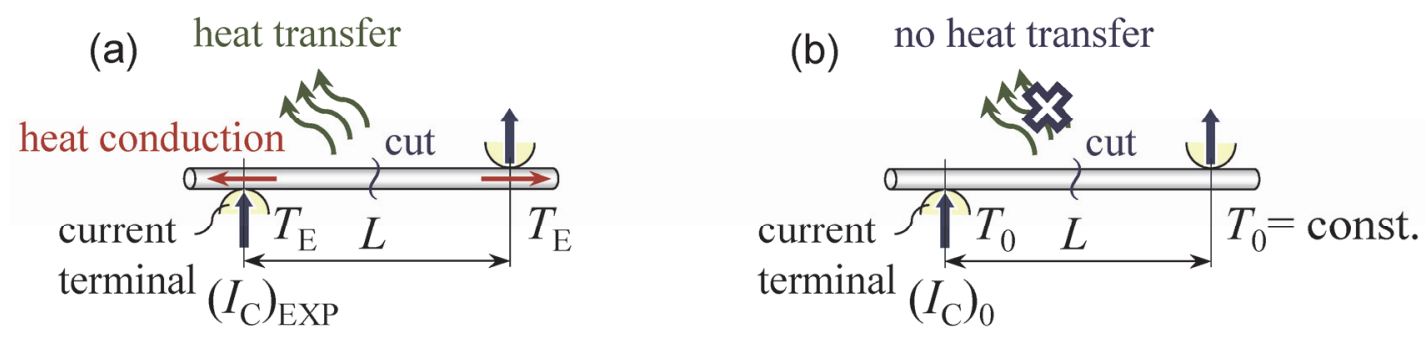

Fig. 1 Two electro-thermal problems. The currents required to cut the wire by (a) experiment, $\left(I_{\mathrm{C}}\right)_{\mathrm{EXP}}$, and (b) analysis, $\left(I_{\mathrm{C}}\right)_{0}$. Here in the analysis, the thermal boundary conditions, where there is no heat transfer from the wire surface, and the temperature at the ends for current supply is constant at the ambient temperature, $T_{0}$, are assumed. 
values of $f$ can be obtained as the function of $L$ by conducting the experiment to cut the wire for various lengths. The values of $f$ greater than unity indicates that the temperature in the wire is easily increased compared with the case under the thermal boundary conditions assumed.

\section{Experiment to cut the wires with Joule heating}

The values of $d$ of the Pt wires offered for the testing were $0.8,5$ and $20 \mu \mathrm{m}$. To determine $f$ values for the wires, the wires were cut with Joule heating under the various values lengths, where the slenderness of the wires $(d / L)$ were 0.002 , $0.003,0.004,0.008$, and 0.016 . Ag terminals of $75 \mu \mathrm{m}$ thickness were used for the current supply. All the experiments were performed in the air $\left(T_{0}=293 \mathrm{~K}\right)$. From $0.1 \mathrm{~mA}$, the supplied current was gradually increased by $0.1 \mathrm{~mA}$, then repeatedly increased the current until the wire was cut. This investigation was conducted five times using the same value of $d / L$ for each diameter, and the average value was used to determine $f$. As shown an example for $d=20 \mu \mathrm{m}$ and $L=$ $1.25 \mathrm{~mm}$ in Fig. 2(a), for all the cases, the wire was cut near the middle for the current supply. The values of $\left(I_{\mathrm{C}}\right)_{\mathrm{EXP}}$ for the wires having $d$ of $0.8,5$ and $20 \mu \mathrm{m}$ are shown in Figs. 2(b) to (d), respectively. For all the cases, $\left(I_{\mathrm{C}}\right)_{\mathrm{EXP}}$ decreases with increase in $L$, and the wire having the greater $d$ requires the larger value of $\left(I_{\mathrm{C}}\right)_{\mathrm{EXP}}$.

The values of $f$ determined from $\left(I_{\mathrm{C}}\right)_{\mathrm{EXP}}$ obtained experimentally, and Eqs. (1) and (2) are shown in Fig. 3. Here the following values of the Pt were used to determine $f: K=72 \mathrm{~W} \mathrm{~m}^{-1} \mathrm{~K}^{-1}, \sigma=9.45 \times 10^{6} \mathrm{~S} \mathrm{~m}^{-1}$, and $T_{\mathrm{M}}=2042 \mathrm{~K}$. At the same value of $d / L$, the values of $f$ for thinner wire were greater than those for thicker one, and this indicates that the temperature of the wire having smaller diameter was easily increased by Joule heating. Moreover, this tendency became remarkable with increasing $d / L$. In Fig. 3, the values of $f$ become less than unity for smaller value of $d / L$. On the other hand, these becomes greater than unity for larger values of $d / L$. Let us consider the reason for this. In case that $L$ is longer, heat transfer from the wire surface becomes notable. This contributes to increase $\left(I_{\mathrm{C}}\right)_{\mathrm{EXP}}$, and $f$ becomes smaller than unity. As described in Appendix, $\left(I_{\mathrm{C}}\right)_{0}$ is determined under the thermal boundary conditions where the temperature at both ends for current supply is constant at $T_{0}$. However, in the realistic situation, the temperature at the ends for current supply becomes greater than $T_{0}$, and this contributes to decrease $\left(I_{\mathrm{C}}\right)_{\text {EXP. }}$ In case that $L$ is shorter, increase in the temperature at both ends for current supply affects $\left(I_{\mathrm{C}}\right)_{\text {EXP }}$ greatly than the effect of heat transfer, and $f$ becomes greater than unity.

We approximate the relationships between $f$ and $d / L$ by the following polynomial equation:

$$
f=\sum_{j=1}^{N} k_{j}\left(\frac{d}{L}\right)^{j}
$$
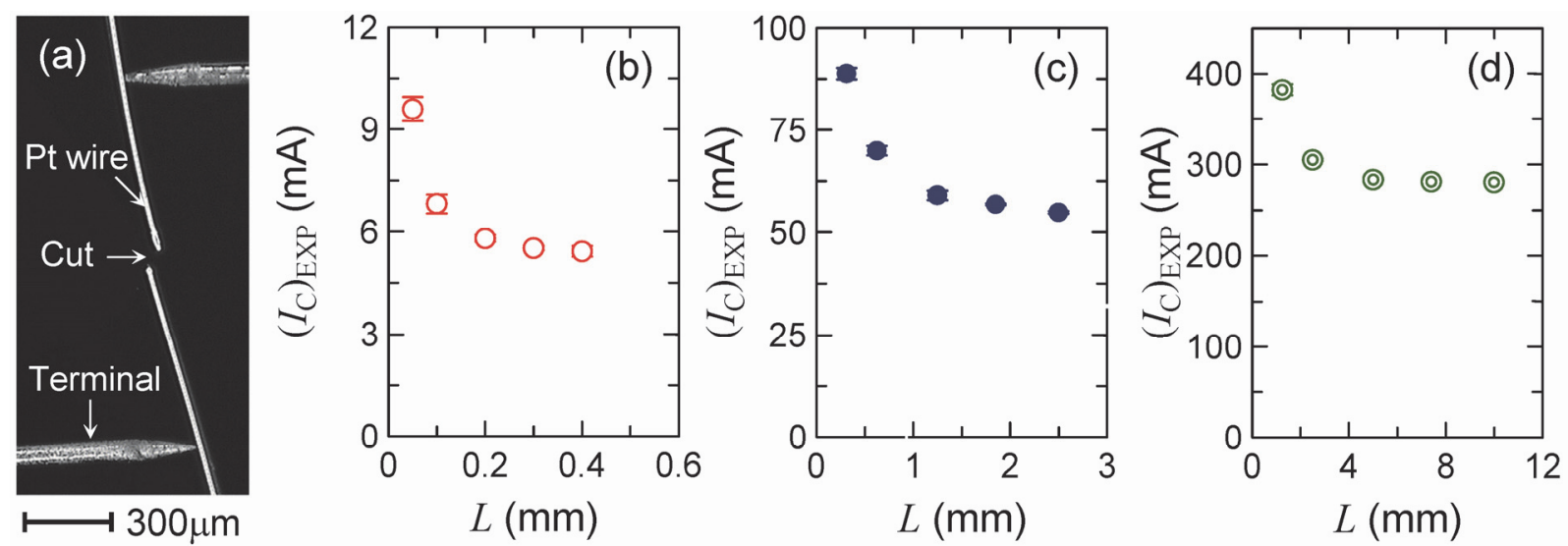

Fig. 2 Experiment for cutting the wires by Joule heating. (a) An example of cutting the wire $(d=20 \mu \mathrm{m}$ and $L=$ $1.25 \mathrm{~mm}$ ), where the wire was cut at the middle of the current supply. (IC)EXP vs. $L$ for (b) $d=0.8 \mu \mathrm{m},(\mathrm{c}) d=5$ $\mu \mathrm{m}$ and (d) $d=20 \mu \mathrm{m}$. 


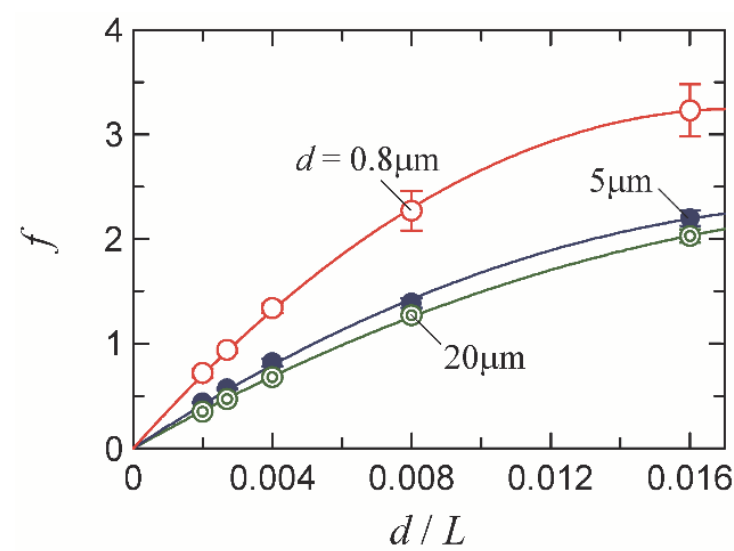

Table 1 Coefficients of $k_{1}$ and $k_{2}$ for $d=0.8,5$ and $20 \mu \mathrm{m}$.

\begin{tabular}{c|c|c|c}
\hline \hline$d(\mu \mathrm{m})$ & $k_{1}$ & $k_{2}$ & $R^{2}$ \\
\hline 0.8 & $3.73 \times 10^{2}$ & $-1.07 \times 10^{4}$ & 0.9997 \\
5 & $2.20 \times 10^{2}$ & $-5.15 \times 10^{3}$ & 0.9985 \\
20 & $1.87 \times 10^{2}$ & $-3.74 \times 10^{3}$ & 0.9997 \\
\hline
\end{tabular}

Fig. $3 f$ vs. $d / L$ for $d=0.8,5$ and $20 \mu \mathrm{m}$.

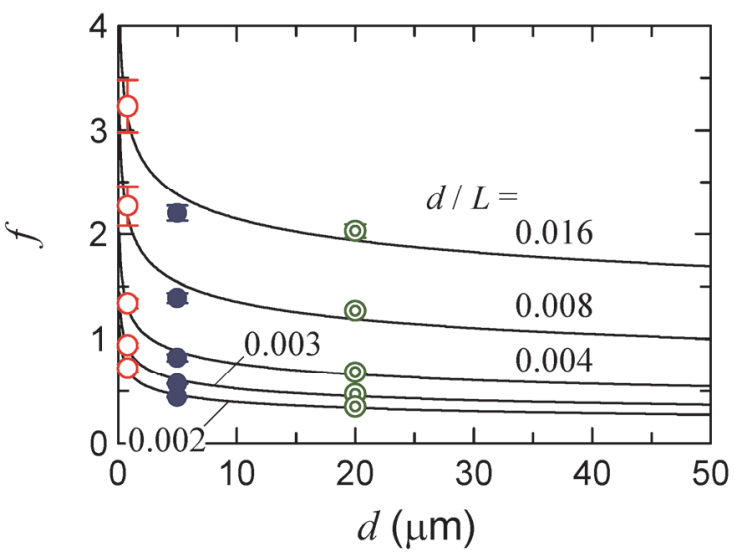

Table 2 Coefficients of $m$ and $n$ for $d=0.8,5$ and $20 \mu \mathrm{m}$.

\begin{tabular}{c|c|c|c}
\hline \hline$d / L$ & $m$ & $n$ & $R^{2}$ \\
\hline 0.002 & $4.13 \times 10^{-2}$ & $-2.28 \times 10^{-1}$ & 0.9883 \\
0.003 & $5.93 \times 10^{-2}$ & $-2.20 \times 10^{-1}$ & 0.9790 \\
0.004 & $9.44 \times 10^{-2}$ & $-2.11 \times 10^{-1}$ & 0.9688 \\
0.008 & $2.15 \times 10^{-1}$ & $-1.86 \times 10^{-1}$ & 0.9108 \\
0.016 & $4.96 \times 10^{-1}$ & $-1.48 \times 10^{-1}$ & 0.9244 \\
\hline
\end{tabular}

Fig. $4 f$ vs. $d$ for $d / L=0.002,0.003,0.004,0.008$ and 0.016 .

In this study, we selected $N=2$, and the coefficients of $k_{1}$ and $k_{2}$ for the wires of $d=0.8,5$, and $20 \mu \mathrm{m}$ are summarized in Table 1. Correlation coefficient, $R^{2}$, for this fitting was close to unity. The values of $k_{1}$ decrease with increase in $d$. On the other hand, the values of $k_{2}$ is increasing in $d$. The difference in the values of $f$ for the wires having the different values of $d$ were brought by the difference in the thermal boundary conditions around the wire.

\section{Prediction of thermal boundary conditions around the wire}

In the previous chapter, it was found that the values of $f$ increased with decrease in $d$ at the same value of $d / L$. In this chapter, let us predict the values of $f$ for the wire having different value of $d$. The values of $f$ are plotted against $d$ in Fig. 4. For a value of $d / L$, th values of $f$ decrease with increase in $d$, and those are saturated at the larger value of $d$. Also, at a value of $d$, larger values of $d / L$ gave the greater values of $f$. The values of $f$ are approximated as a function of $d$ by the following function:

$$
f=m\left(\frac{d}{d_{0}}\right)^{n}
$$

where $m$ and $n$ are the dimensionless constants, and $5 \mu \mathrm{m}$ was selected as $d_{0}$. The values of $m$ and $n$ are summarized in Table 2 together with the coefficient of correlation. The values of $m$ and $n$ increase with increase in $d / L$. $R^{2}$ for this fitting was above 0.9 , and the behavior was reasonably fitted by the exponential function. 


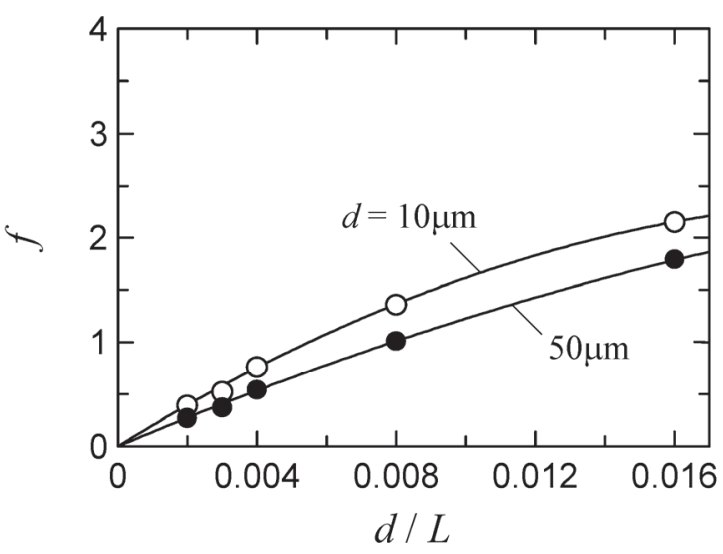

Table 3 Coefficients of $k_{1}$ and $k_{2}$ for $d=10$ and $50 \mu \mathrm{m}$.

\begin{tabular}{c|c|c|c}
\hline \hline$d(\mu \mathrm{m})$ & $k_{1}$ & $k_{2}$ & $R^{2}$ \\
\hline 10 & $2.07 \times 10^{2}$ & $-4.53 \times 10^{3}$ & 0.9999 \\
50 & $1.41 \times 10^{2}$ & $-1.84 \times 10^{3}$ & 0.9999 \\
\hline
\end{tabular}

Fig. 5 Predicted values of $f$ for $d=10$ and $50 \mu \mathrm{m}$.
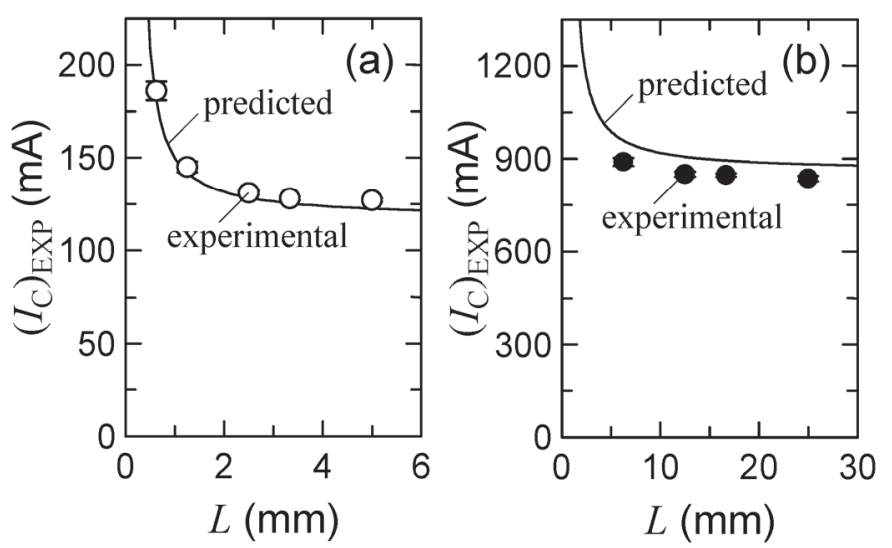

Fig. 6 Predicted and experimental values of $\left(I_{\mathrm{C}}\right)_{\mathrm{EXP}}$ for (a) $d=10 \mu \mathrm{m}$ and (b) $d=50 \mu \mathrm{m}$. Here the predicted values were determined from Eqs. (1) and (2) with use of the values of $f$ shown in Fig. 5.

Using Eq. (4), let us predict the values of $f$ for the wires of $d=10$ and $50 \mu \mathrm{m}$. From Eq. (4), the values of $f$ at $d / L=$ $0.002,0.003,0.004,0.008$, and 0.016 were determined, and the values of $f$ for $d=10$ and $50 \mu \mathrm{m}$ are plotted in Fig. 5. By fitting the data, the values of $k_{1}$ and $k_{2}$ in Eq. (3) for $d=10$ and $50 \mu \mathrm{m}$ were determined, and the those are summarized in Table 3. The values of $R^{2}$ was close to unity. Predicted values of $f$ were used to determine $\left(I_{\mathrm{C}}\right)_{\mathrm{EXP}}$ from Eqs. (1) and (2), and the values of $\left(I_{\mathrm{C}}\right)_{\mathrm{EXP}}$ for the wires having $d=10$ and $50 \mu \mathrm{m}$ are shown in Figs. 6(a) and (b), respectively. The experiment to cut the wire of $d=10 \mu \mathrm{m}$ was performed for $L=0.625,1.25,2.50,3.33$ and $5.00 \mathrm{~mm}$, and the wire of $d=$ $50 \mu \mathrm{m}$ was cut at $L=6.25,12.5,16.6$ and $25.0 \mathrm{~mm}$. The values of $\left(I_{\mathrm{C}}\right)_{\text {EXP }}$ decreases with increase in $L$, and the predicted values of $\left(I_{\mathrm{C}}\right)_{\mathrm{EXP}}$ were in good agreement with the experimental ones for both cases. Especially, the differences between the predicted and experimental values for the case of $d=10 \mu \mathrm{m}$ [Fig.6 (a)] were smaller than those of $d=50 \mu \mathrm{m}$ [Fig.6 (b)].

\section{Experiment to join the wires with Joule heating}

To confirm the validity of the determined values of $f$, two Pt wires were joined together with Joule heating. The tips of two wires were contacted together, and the constant direct current was supplied to the wires. From $0.1 \mathrm{~mA}$, the current was gradually increased by $0.1 \mathrm{~mA}$ as the same way to cutting experiment, and repeated increasing the current until the wires were welded. It has been reported that the lower limit for the welding current exists, and by supplying the current for lower limit, the contact of two wires first melts. From the seeming analogy between the melting phenomenon at the contact in the present electro-thermal problem and the crack propagation phenomenon in the fracture mechanics, with referring the stress intensity factor, a governing parameter for the melting phenomenon at the contact by Joule heating has been proposed, and is given by (Tohmyoh, 2009) 


$$
U=\frac{I}{A} L f
$$

The condition for joining two wires is expressed by $U_{\mathrm{L}}<U<U_{\mathrm{U}}$, where $U_{\mathrm{L}}$ and $U_{\mathrm{U}}$ are the lower and upper limits for the joining. It has been experimentally investigated that the value of $U_{\mathrm{L}}$ is about $70 \%$ of $U_{\mathrm{U}}$.

In the experiment, the contact was located at the middle of $L$, and the current was increased by $0.1 \mathrm{~mA}$ until the wires were first confirmed to be joined. The lower limit of the current to join the wires was denoted as $I_{\mathrm{L}}$. Next, the current was increased until the joined wires were cut due to over Joule heating, and the upper limit of the current was denoted as $I_{\mathrm{U}}$. The experiment to weld the wires of $d=0.8 \mu \mathrm{m}$ was performed for $L=0.14,0.28$, and $0.35 \mathrm{~mm}$, and that of $d=5 \mu \mathrm{m}$ was for $L=0.4,0.8$, and $1.6 \mathrm{~mm}$. The values of $L$ of $d=20 \mu \mathrm{m}$ were 3,6 , and $9 \mathrm{~mm}$. The values of $I_{\mathrm{L}}$ and $I_{\mathrm{U}}$ for the Pt wires having $d$ of $0.8,5$, and $20 \mu \mathrm{m}$ are shown in Fig. 7(a) to (c), respectively. For all the cases, the values of $I_{\mathrm{L}}$ and $I_{\mathrm{U}}$ decrease with increase in $L$, similarly to the behavior of $\left(I_{\mathrm{C}}\right)_{\text {EXP }}$ shown in Fig. 2.

With considering the difference in the thermal boundary conditions around the wires, let us summarize the condition of Joule heat welding of the wires. The values of $U$ determined from $I_{\mathrm{L}}$ and $I_{\mathrm{U}}$ for all the wires were plotted against $d / L$ in Fig. 8. Without depending on $d$, the lower limit of $U, U_{\mathrm{L}}$, and the upper limit of $U$, $U_{\mathrm{U}}$, were found to be coincident with each other. The values of $U_{\mathrm{L}}$ and $U_{\mathrm{U}}$ were $2.2 \times 10^{6}$, and $3.1 \times 10^{6} \mathrm{~A} \mathrm{~m}^{-1}$, respectively. These values were close to those determined previously for the $0.8 \mu \mathrm{m}$ thick Pt wire (Tohmyoh, 2009). Moreover, the wires of $d=10 \mu \mathrm{m}$ were welded together by supplying the currents correspond to $U=2.65 \times 10^{6} \mathrm{~A} \mathrm{~m}^{-1}\left[=\left(U_{\mathrm{U}}+U_{\mathrm{L}}\right) / 2\right]$. The values of $L$ were 1 , 3 and $4 \mathrm{~mm}$, and the corresponding currents were 139, 121 and $115 \mathrm{~mA}$, respectively. For all the cases, two wires were successfully welded together. Finally, the validity and the usefulness of the thermal boundary conditions determined were verified though the welding experiment.
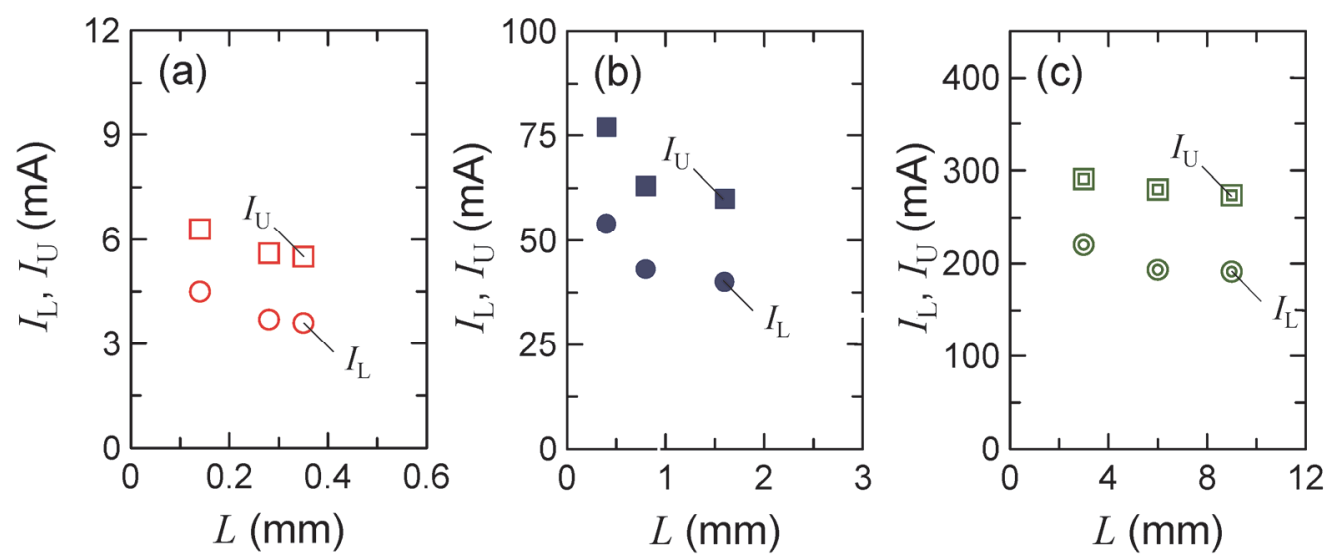

Fig. $7 I_{\mathrm{L}}$ and $I_{\mathrm{U}}$ for (a) $d=0.8 \mu \mathrm{m}$, (b) $d=5 \mu \mathrm{m}$ and (c) $d=20 \mu \mathrm{m}$.

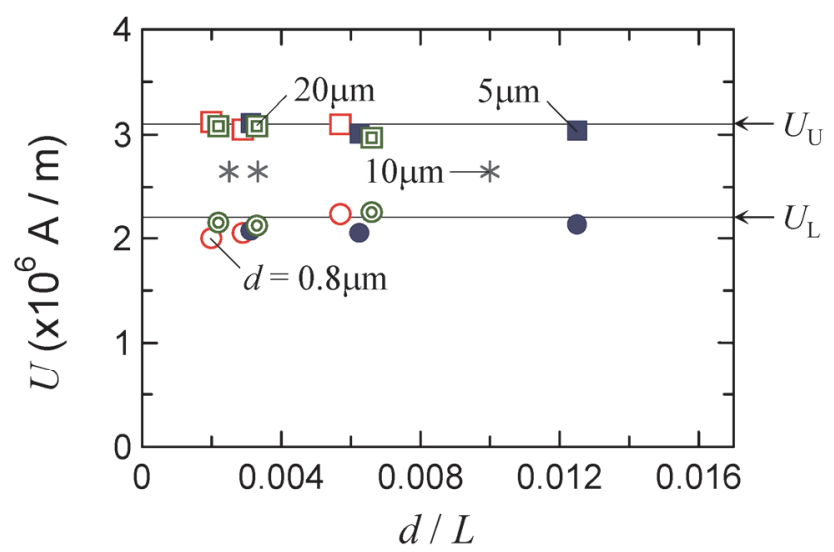

Fig. $8 U$ vs. $d / L$ for $d=0.8,5,10$ and $20 \mu \mathrm{m}$. Here welding the wires of $d=10 \mu \mathrm{m}$ was performed by supplying the currents correspond to $U=2.65 \times 10^{6} \mathrm{~A} \mathrm{~m}^{-1}\left[=\left(U_{\mathrm{U}}+U_{\mathrm{L}}\right) / 2\right]$. 


\section{Conclusions}

In this paper, the thermal boundary conditions around thin Pt wires under the current supply were investigated by conducting the experiments to cut the wires by Joule heating. The diameters of the Pt wires were $0.8,5$, and $20 \mu \mathrm{m}$. The thermal boundary conditions around the wire were affected by the heat transfer from the wire surface to the ambient and the heat conduction at the ends for the current supply. In this study, the thermal boundary conditions were described as the ratio of the current required to cut the wire experimentally to the current required to cut the wire under the assumption that there was no heat transfer from the wire surface and the temperature at both ends for current supply was constant at ambient temperature. It was clear from the changes in the index that the temperature of thinner wire was easily raised due to Joule heating compared with the thicker one. The behavior of the index was found to be approximated by the exponential function against the diameter, and with use of those relations, thermal boundary conditions around the wire having various diameters were predicted. The currents required to cut the wires having the diameter of 10 and $50 \mu \mathrm{m}$ were investigated, and the experimental values were in good agreement with the predicted ones. Moreover, the experiment to join two wires having various diameters were also conducted, and the validity of the thermal boundary conditions around the wires having various diameter, which were determined experimentally or predicted analytically, was verified.

\section{Acknowledgements}

The authors would like to acknowledge Professor M. Saka for valuable discussions throughout this work. This work was supported by JSPS KAKENHI Grant Number $18 \mathrm{H} 01331$.

\section{Appendix}

Let us consider the electro-thermal problem, where the constant direct current is supplied to a thin wire with a pair of the current terminals, see Fig. 1(a). If we assume that the wire is so thin that the temperature is uniform at all points of the cross section, this problem is governed by the following one dimensional differential equation (Carslaw and Jaeger, 1959).

$$
\frac{\partial T}{\partial t}=\kappa \frac{\partial^{2} T}{\partial x^{2}}-v\left(T-T_{0}\right)+\frac{q}{\rho c},
$$

where $T$ is the temperature and is a function of time, $t$, and the position in the wire, $x$. The $x$ axis is in the direction of the wire length, and its origin is located at the left end for the current supply. The symbol $\rho$ is density, $c$ specific heat, $\kappa=K$ $/(\rho c), v=H p /(\rho c A), H$ surface conductance, $p$ perimeter, and $q=I^{2} /\left(A^{2} \sigma\right)$. In case that there is no heat transfer from the wire surface, the second term of the right hand side of Eq. (A1) can be ignored. The steady state temperature for this situation can be determined by setting $\partial T / \partial t=0$ in Eq. (A1) and with the following boundary conditions: $T=T_{0}$ at $x=$ 0 and $L$. We get

$$
T_{1}=\frac{q}{2 K}\left(L x-x^{2}\right)+T_{0}
$$

The temperature of the wire, $T_{1}$, takes its maximum value at the middle of two current terminals. $\left(I_{\mathrm{C}}\right)_{0}$ expressed by Eq. (2) is the value of $I$ obtained by substituting $x=L / 2$ and $T_{1}=T_{\mathrm{M}}$ into Eq. (A2).

\section{References}

Carslaw, H. S. and Jaeger, J. C., Conduction of Heat in Solids, 2nd Ed. (1959), Oxford University Press, London.

Dai, S., Li, Q., Liu, G., Yang, H., Yang, Y., Zhao, D., Wang, W. and Qiu, M., Laser-induced single point nanowelding of silver nanowires, Applied Physics Letters, Vol.108 (2016), 121103, 5 pages.

Dong, L., Tao, X., Zhang, L., Zhang, X. and Nelson, B. J., Nanorobotic spot welding: controlled metal deposition with 
attogram precision from copper-filled carbon nanotubes, Nano Letters, Vol.7 (2007), pp.58-63.

Fujimori, M. and Tohmyoh, H., Welding thin Pt wires with dissimilar diameters by Joule heating, Japanese Journal of Applied Physics, Vol.52 (2013), 06GH01, 4 pages.

Fukui, S. and Tohmyoh, H., Tip to side welding of ultrathin Pt wires by Joule heating, Japanese Journal of Applied Physics, Vol.50 (2011), 057201, 4 pages.

Garnett, E. C., Cai, W., Cha, J. J., Mahmood, F., Connor, S. T., Christoforo, M. G., Cui, Y., McGehee, M. D. and Brongersma, M. L., Self-limited plasmonic welding of silver nanowire junctions, Nature Materials, Vol.11 (2012), pp.241-249.

Hirayama, H., Kawamoto, Y., Ohshima, Y. and Takayanagi, K., Nanospot welding of carbon nanotubes, Applied Physics Letters, Vol.79 (2001), 1169, 3 pages.

Hu, A., Peng, P., Alarifi, H., Zhang, X. Y., Guo, J. Y., Zhou, Y. and Duley, W. W., Femtosecond laser welded nanostructures and plasmonic devices, Journal of Laser Applications, Vol.24 (2012), 042001, 7 pages.

Hummelgård, M., Zhang, R., Carlberg, T., Vengust, D., Dvorsek, D., Mihailovic, D. and Olin, H., Nanowire transformation and annealing by Joule heating, Nanotechnology, Vol.21 (2010), 165704, 6 pages.

Jin, C., Suenaga, K. and Iijima, S., Plumbing carbon nanotubes, Nature Nanotechnology, Vol.3 (2007), pp.17-21.

Kim, S. J. and Jang, D.-J., Laser-induced nanowelding of gold nanoparticles, Applied Physics Letters, Vol. 86 (2005), 033112, 3 pages.

Lu, Y., Huang, J. Y., Wang, C., Sun, S. and Lou, J., Cold welding of ultrathin gold nanowires. Nature Nanotechnology, Vol.5 (2010), pp.218-224.

Ma, Y., Li, H., Bridges, D., Peng, P., Lawrie, B., Feng, Z. and Hu, A., Zero-dimensional to three-dimensional nanojoining: current status and potential applications, RSC Advances, Vol.6 (2016), pp.75916-75936.

Peng, Y., Cullis, T. and Inkson, B., Bottom-up nanoconstruction by the welding of individual metallic nanoobjects using nanoscale solder, Nano Letters, Vol.9 (2009), pp.91-96.

Peng, P., Liu, L., Gerlich, A. P., Hu, A. and Zhou, Y. N., Self-oriented nanojoining of silver nanowires via surface selective activation, Particle \& Particle Systems Characterization, Vol.30 (2013), pp.420-426.

Song, T.-B., Chen, Y., Chung, C.-H., Yang, Y. M., Bob, B., Duan, H.-S., Li, G., Tu, K.-N., Huang, Y. and Yang, Y., Nanoscale Joule heating and electromigration enhanced ripening of silver nanowire contacts, ACS Nano, Vol.8 (2014), pp.2804-2811.

Tan, Y. and Wang, Y.-G., Current density-sensitive welding of a semiconductor nanowire to a metal electrode, Chinese Physics Letters, Vol.30 (2013), 017901, 5 pages.

Tohmyoh, H., Imaizumi, T., Hayashi, H. and Saka, M., Welding of Pt nanowires by Joule heating, Scripta Materialia, Vol.57 (2007), pp.953-956.

Tohmyoh, H., A governing parameter for the melting phenomenon at nanocontacts by Joule heating and its application to joining together two thin metallic wires, Journal of Applied Physics, Vol.105 (2009), 014907, 9 pages.

Tohmyoh, H. and Fukui, S., Self-completed Joule heat welding of ultrathin Pt wires, Physical Review B, Vol.80 (2009), 155403, 7 pages.

Tohmyoh, H. and Fujimori, M., Joule heat welding of thin wires to thin films, Mechanical Engineering Journal, Vol.1 (2014), 13-00221, 10 pages.

Tohmyoh, H. and Sunagawa, T., A parameter governing the melting induced at the micrometer level in a dissimilar metal wire system by Joule heating, Journal of Applied Physics, Vol.117 (2015), 234901, 6 pages.

Vafaei, A., Hu, A. and Goldthorpe, I. A., Joining of individual silver nanowires via electrical current, Nano-Micro Letters, Vol.6 (2014), pp.293-300.

Xu, S., Tian, M., Wang, J., Xu, J., Redwing, J. M. and Chan, M. H. W., Nanometer-scale modification and welding of silicon and metallic nanowires with a high-intensity electron beam, Small, Vol.1 (2005), pp.1221-1229. 